\title{
Mind the gap: a step forward in supporting hospice-based research
}

\section{Anthony Byrne, ${ }^{1}$ Laura Upton, ${ }^{2}$ Sarah Townsend ${ }^{2}$}

The late Nobel laureate Seamus Heaney used a geological analogy of igneous and sedimentary rock formation to describe modes of poetic composition. ${ }^{1}$ Igneous rocks, derived from solidified magma, imply an eruptive, pre-emptory form of composition. Sedimentary rock formation in contrast is by accrual. It strikes us that such analogy fits equally well for the evolution of clinical research ideas: sometimes rapid or unlooked for, more often driven by observation and recognition of patterns over time. Today for most of us, in reality, the analogy sadly all too often evokes the weather-beaten image of the wearing down rather than the building up.

Anyone familiar with the drama of transforming a research project from idea to fruition may be forgiven for feeling that the Atlantic ocean has indeed washed over them more than once. Papers continue to appear in the palliative care literature documenting the many challenges, with familiar themes over time. ${ }^{2} 3$ Common among these are excessive bureaucracy and lack of clinician time, funding and institutional capacity. $^{4} 5$

Progress has been made, with focused efforts to improve funding and infrastructure, patient recruitment, ${ }^{6}$ and to address methodological challenges. ${ }^{7}$ A recent Lancet editorial has also highlighted the current contexts which make more

\footnotetext{
${ }^{1}$ Marie Curie Palliative Care Research Centre, School of Medicine, Cardiff University, Cardiff, UK: ${ }^{2}$ R\&D Department, Velindre NHS Trust, Cardiff, UK

Correspondence to Dr Anthony Byrne, Marie Curie Palliative Care Research Centre, School of Medicine, Cardiff University, Cardiff, UK; Anthony.Byrne2@wales.nhs.uk
}

palliative research imperative for informing practice. ${ }^{8}$

Gaps undoubtedly remain. Ironically, the environment which drives the need for evidence of efficacy and efficiency-a competitive and stretched healthcare and social care resource-is in itself a significant threat. Service delivery pressures remain a substantial obstacle for multidisciplinary clinicians, where research is seen as an additional time burden rather than an integral part of care. The perverse incentive is to deliver services/interventions at volume rather than dynamically assess and ensure effectiveness.

Of particular importance in this respect is research as process: not simply methodologically in terms of design, but practically in terms of delivery. Care settings may define a patient's or carer's ability to access research of relevance to them. Similarly robust outcomes are predicated on robust data capture, which requires a flexible and innovative approach to following patients across settings, undertaking assessments in places of their choice. So the need for a healthy research culture in hospices, including independent settings, is defined.

A clearly identified gap here is a hospice organisation's understanding of research practicalities and procedures. Although well honed in the craft of clinical governance, independent hospices may baulk at the notion of research governance, robbed as they are of NHS Research and Development (R\&D) insight and support. We have surveyed independent palliative care settings in Wales and found enthusiasm for undertaking research dampened by lack of knowledge, lack of resources and concerns about financial and clinical impact-resonating with international findings elsewhere. ${ }^{5} 9$ We therefore proposed the development of guidance in the form of a research governance toolkit for independent hospices, a concept that was universally endorsed in our survey.

The aim of the toolkit is to provide guidance on host organisation roles and responsibilities, improving knowledge and minimising workload for staff. In other words, it does not tell you how to design a research project, but how to accommodate and facilitate it. A key concern for independent hospices is the potential opportunity cost to patient and family clinical support of engaging in research. Using concise and accessible information, the toolkit aims to minimise redirection of resources by identifying others and by clarifying processes. Fostering supportive relationships between clinical and research staff allows each to understand and respect the other's workload and role in the clinical field. Improved knowledge can also improve the quality of organisational debate around the importance of research and evaluation ${ }^{10}$ and resolving those resource tensions. Engaging that level of reflexivity has positive implications for all aspects of hospice activity.

The toolkit has been developed as a web-based resource to improve accessibility: http://www.nischrcancerrrg.org/research/. Although developed for Wales, it is equally relevant to practice across the UK. It has three layers to its guidance: an overview layer which outlines what needs to be done, why and how (figure 1), which directly links to a second and then a third layer with increasing levels of information and access to source documents. Staff can choose which level suits them best for a particular task. In the 'how to' section there is practical guidance on issues such as training (with links to regional training programmes), resource use and audit trails, and an optional template for risk review which can 


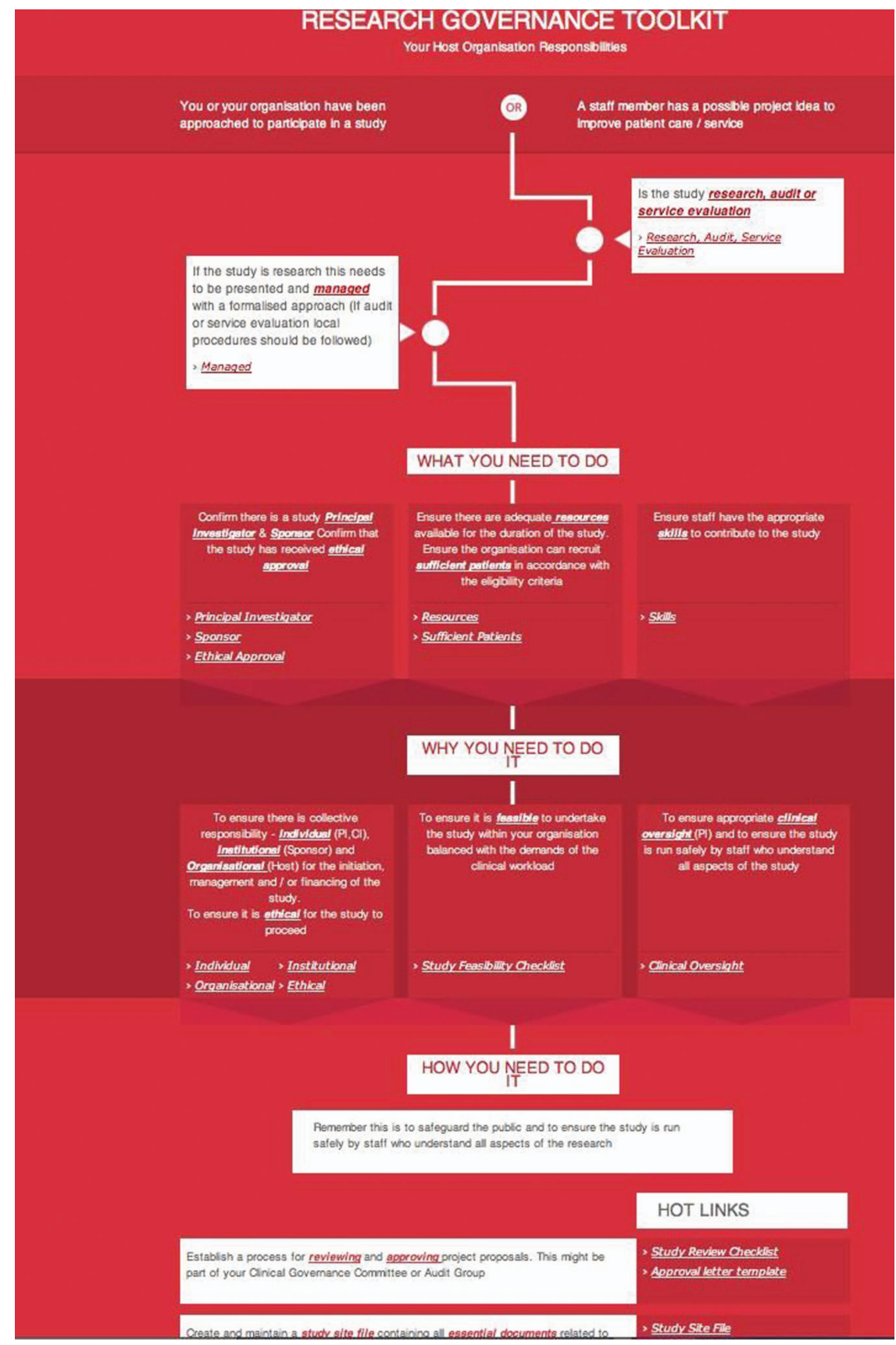

Figure 1 The overview page of the Research Governance Toolkit for Independent Hospices.

be incorporated into usual governance activities.

The toolkit has been developed using an iterative process, led by the authors but supported by collaborations from the National Institute for Social Care and Health Research (NISCHR) in Wales, the Wales Cancer Trials Unit, Velindre NHS Trust: a highly research active Cancer Centre, and the Marie Curie Palliative Care Research Centre at Cardiff University. A draft version was reviewed by a range of hospice staff from across
Wales and feedback on content, structure and wording informed the final version. It is therefore structured to be comprehensive but user-friendly and practically focused.

By encouraging its use we hope to develop the conversation, in Wales and beyond, between independent and statutory sectors around research mentorship and support. By demystifying research governance we also hope to empower boards of Trustees, managers and clinical staff to inform the debate on the importance of a research active culture across all care settings. ${ }^{11}$ User feedback via the website is welcomed to further develop and improve the resource.

Acknowledgements The authors

acknowledge the input and support of independent hospices in Wales, as well as the support of NHS R\&D offices.

Contributors $\mathrm{AB}$ developed the original idea and subsequently developed the toolkit alongside $\mathrm{LU}$ and $\mathrm{ST}$. $\mathrm{AB}$ wrote the manuscript with contributions from LU and ST.

Funding This project was in part funded by a NISCHR Academic Health Sciences Collaboration Fellowship (AB). 


\section{Competing interests None.}

Provenance and peer review Not commissioned; externally peer reviewed.

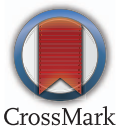

To cite Byrne A, Upton L, Townsend S. BMJ Supportive \& Palliative Care 2015;5:4-6.

Received 23 October 2014

Revised 18 January 2015

Accepted 31 January 2015

\section{REFERENCES}

1 Heaney S. Finders keepers: selected prose 1971-2001. London, UK: Faber and Faber Ltd, 2002.

2 Casarett D, Karlawish J, Hirschman K. Are hospices ready to participate in palliative care research? Results of a national survey. J Palliat Med 2002;5:397-406.
3 Sigurdardottir KR, Haugen DF, van der Rijt CCD, et al. Clinical priorities, barriers and solutions in end-of-life cancer care research across Europe. Report from a workshop. Eur J Cancer 2010;46:1815-22.

4 Rhondali W, Berthiller J, Hui D, et al. Barriers to research in palliative care in France. BMJ Support Palliat Care 2014;4:182-9.

5 Chen EK, Riffin C, Reid CM, et al. Why is high-quality research on palliative care so hard to do? Barriers to improved research from a survey of palliative care researchers. J Palliat Med 2014;17:782-7.

6 NCRI Palliative and Supportive Care Clinical Studies Group Annual Report 2012/2013. London: National Cancer research Institute (NCRI), 2013.

7 Currow DC. The PRISMA Symposium 3: lessons from beyond Europe. Why invest in research and service development in palliative care? An Australian perspective. J Pain Symptom Manage 2011;42:505-10.

8 [No authors listed]. Palliative care: more than one chance to get it right. Lancet 2014;384:103.

9 Sigurdardottir KR, Haugen DF, Bausewein $\mathrm{C}$, et al. A pan-European survey of research in end-of-life cancer care. Support Care Cancer 2012;20:39-48.

10 Duke S, Bennett H. A narrative review of the published ethical debates in palliative care research and an assessment of their adequacy to inform research governance. Palliat Med 2010;24:111-26.

11 Gysels M, Evans CJ, Lewis P, et al. MORECare research methods guidance development: recommendations for ethical issues in palliative and end-of-life care research. Palliat Med 2013;27:908-17. 